\title{
Development of a Wireless Inertial Measurement System for Pitching Motion Analysis
}

\author{
Seiji Kitamura, a , Koichi Sagawa ${ }^{1, b}$, Toshiaki Tsukamoto ${ }^{2}$, Yasuyuki Ishibashi ${ }^{3}$ \\ ${ }^{1}$ Dept. of Intelligent Machines and System Engineering, Hirosaki University, Aomori, Japan \\ ${ }^{2}$ Hirosaki University School of Medicine \& Hospital, Aomori, Japan \\ ${ }^{3}$ Graduate School of Medicine and School of Medicine, Hirosaki University, Aomori, Japan \\ ah09gs851@stu.hirosaki-u.ac.jp, bsagawa@cc.hirosaki-u.ac.jp
}

Keywords: Pitching, inertial measurement, acceleration, angular velocity, wireless sensor

\begin{abstract}
This paper presents a wireless inertial measurement system to analyze three- dimensional (3D) pitching movement of baseball. Developed wireless inertial measurement unit (WIMU) sizes $43.7 \times 45.2 \times 25.7[\mathrm{~mm}]$, weighs 48 [g] including Lithium-Ion battery, and consists of two types of 3D accelerometers, two types of 3D gyroscopes, release sensor, a microcontroller (MCU), flash memory, and an RF module. Synchronization of start and completion of measurement procedure for plural WIMUs are wirelessly controlled by a host computer. Three-dimensional pitching form of upper limb and trunk is produced by the numerical integration of the acceleration and angular velocity. The experimental results show that 3D posture, trajectory and joint torque in overhand and sidearm throwing are successfully estimated using the proposed system.
\end{abstract}

\section{Introduction}

One of the reasons of throwing injuries such as pitching elbow and pitching shoulder is the overuse of upper arm with inappropriate pitching movement [1]. Therefore, it is necessary to coach a player appropriate pitching movement. The question is the method how to find the inappropriate movement in high-speed movement such as pitching. High-speed movement is acquired using optical motion capture system recently. This system enables precise measurement of body landmark but has some disadvantages such as limitation of measurement place and high cost of employment. To resolve this problem, inertial measurement unit (IMU) which consists of accelerometers and gyroscopes has been proposed recently. In the previous study the authors proposed the estimation method of 3D trajectory of trunk, shoulder, elbow and wrist using three wired IMUs, which sizes $40 \times 55 \times 23[\mathrm{~mm}]$ and weighs 50 [g] without signal cable, mounted on upper arm, forearm, and trunk. Serious consideration of the sensor installation to the upper limbs with respect to the sensor axis and longitudinal direction of the limbs is not required because the relation between installed sensor and upper limb is determined by performing predetermined motions. However, connecting cables between sensors and host computer are obstructive to the pitching movement and prevents the IMU from applying to the patients at the medical site. Therefore, wireless inertial measurement unit applicable to the fast motion is required.

The wireless IMU (WIMU) developed in this paper consists of two types of 3D accelerometers, two types of 3D gyroscopes, release sensor, a microcontroller, removable flash memory, and an RF module. The WIMU acquires 12 bit binary data from 13 analog input channels with sampling frequency of $1000[\mathrm{~Hz}]$ and stores to the memory card. In this paper, details of the WIMU for 3D motion analysis of pitching movement of baseball and its application to visualize two kinds of 3D pitching motions are introduced.

\section{Methods}

Hardware structure. Due to 13 channels input for sensors with 2 bytes each and $1[\mathrm{kHz}]$ of sampling frequency in addition to the requirement of the multi-point measurement, wired data acquisition system that the authors have been proposed measures analog data using thick and parallel cables to acquire the output of the inertial sensors. However, cabling between the sensor and the data logger 


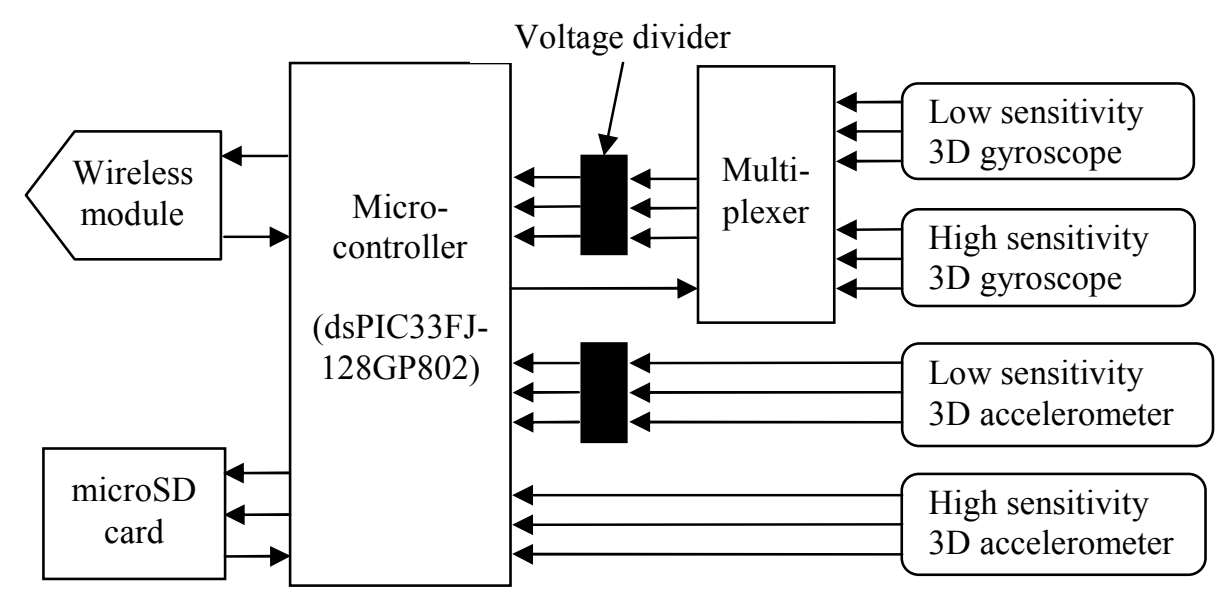

Fig. 1. Block diagram of WIMU.

obstructs the movement of the player. Considering the data transfer of the measured binary data instead of the analog data, the transfer speed requires faster than 200[kbps] per one IMU. Therefore it is difficult to transmit the measured data to the data storage system in real time from plural inertial sensor using current wireless communication technique, although wireless transmission of the measured data solves the interference of the connection cable.

In this paper, therefore, each WIMU is equipped with the data storage memory using microSD card and the control feature of the WIMU such as the initiation and the completion of the data sampling can be performed by wireless communication from a host computer. Moreover, to prevent the WIMU from interfering with the performance of the player, the WIMU is as small and light as possible. A 16-bit microcontroller (dsPIC33FJ128GP802, MICROCHIP) with the 28-pin QFN-S package $(6 \times 6 \times 0.9[\mathrm{~mm}])$ is used for the data acquisition, processing and storage. It has 16 [Kbyte] RAM, 10-channel 12bit AD converter and SPI port which is used for data storage to microSD card. Fig. 1 illustrates the schematic diagram of the WIMU. A triple 2-channel multiplexer (Toshiba, TC4053BFT) is employed to increase AD inputs to 13 channels which provide 6 channels for accelerometer, 6 channels for gyroscope and 1 channel for a release sensor. Two types of accelerometer are used to achieve a wide dynamic range measurement. A 3-axis high sensitivity accelerometer with full-scale range of \pm 11 [G] (MMA7340L, Freescale Semiconductor) and three 1 -axis low sensitivity accelerometers with full-scale range of \pm 120 [G] (ADXL193, Analog Devices) are selected for the measurement of the acceleration. The axes of the low sensitivity accelerometers are located perpendicular each other and their outputs are processed for the motion analysis when the absolute value of the generated acceleration exceeds $\pm 11[\mathrm{G}]$. Similar to the accelerometer, two types of gyroscopes with different dynamic range are used to achieve a wide dynamic range of measurement. Three 1-axis high sensitivity gyroscopes with full-scale range of \pm 300 [deg/s] (ENC-03R, Murata) are mounted perpendicular each other. Two 2-axis low sensitivity gyroscopes with full-scale range of \pm 6000 [deg/s] (LPR5150AL and LPY5150AL, STMicroelectronics) are also installed to measure three-dimensional angular velocity. The former (LPR5150AL) is used to measure the pitch and roll angular velocity while the latter (LPY5150) measures yaw angular velocity only. The output from the low sensitivity gyroscope is processed when absolute value of angular velocity is larger than $\pm 300[\mathrm{deg} / \mathrm{s}]$.

Wireless communication module (XBee, MaxStream) which is compatible with Zigbee protocol is employed for wireless operation from the host computer to synchronize the start and quit of the data acquisition for plural WIMUs. Data transmission speed is set to 115,200 [bps]. As shown in Fig. 2, the WIMU developed in this study sizes $43.7 \times 45.2 \times 25.7[\mathrm{~mm}]$ and weighs 48 [g] including Lithium-Ion battery (7.4[V]). After the measurement of the pitching motion, microSD card is removed from the WIMU and is insterted to the computer to analyze the $3 \mathrm{D}$ pitching motion. 


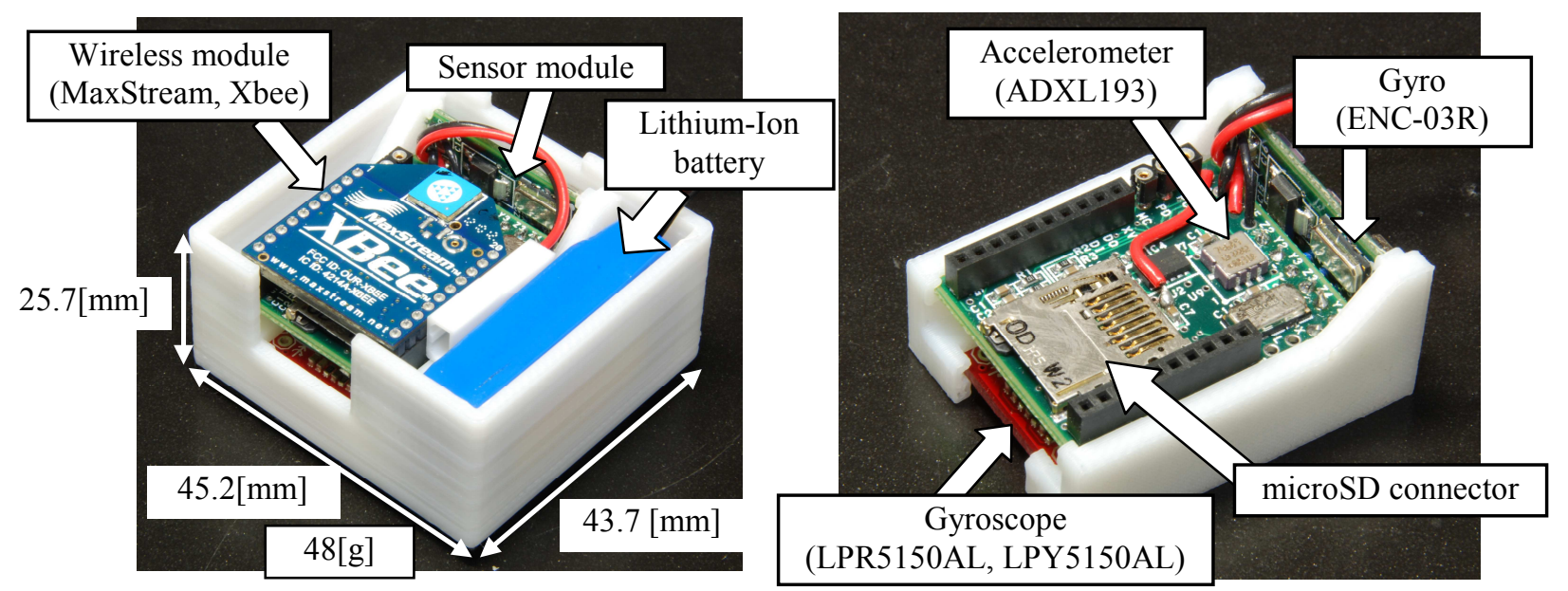

(a) Overview of the WIMU

(b) Sensor module

(Wireless module is removed.)

Fig. 2. (a) Overview of the wireless inertical measuremnt unit (WIMU). Microcontroller, high sensitive accelerometer and multiplexer are mounted backside of the circuit board of the sensor module. (b) Sensor module located under the wireless module shown in the left figure.

Firmware structure. File I/O Functions Using Microchip's Memory Disk Drive File System Library provided from Microchip Technology is used to write measured data to a microSD card. Because of an individual difference of the initialization procedure for microSD cards, processing time of a file open function is variable among microSD cards. Therefore, the file open function must be finished before receiving a synchronization signal from the host computer to start measurement routine. The WIMU starts the measurement procedure when it receives a synchronization start signal.

Next, the structure how to acquire 13 channel data using only 10 channels AD inputs is explained. The multiplexer (MUX) is connected to six sensors and provides outputs from three sensors based on the state of the signal selection port. Firstly, the WIMU acquires analog data from channel 1 to 10; directly from 7 channels and 3 channels through the MUX. Secondly, the microcontroller changes the signal selection level for the MUX and acquires from channel 8 to 10 to obtain three outputs from remaining sensors. Finally, it is possible to achieve 13-channel acquisition by using the MCU with 10 channel analog input.

The WIMU has two temporary storage buffers to retain high speed record of the measured data to the microSD card because the data writing procedure to the microSD card must be performed every 512 bytes. When one buffer memory is filled with the measured data, the stored data is written to the microSD card, and the other buffer memory is used to store the next measured data.

When the WIMUs receive a stop signal, they finish the measurement procedure.

Estimation algorithm. Three WIMUs developed are used to estimate the 3D trajectory of forearm, upper arm and trunk and the joint torque of shoulder and elbow. Acceleration and angular velocity measured on the WIMU coordinate system are transformed to the acceleration and angular velocity based on the global coordinate system using the frame matrix which indicates the posture of the WIMU. Initial frame matrix is calculated by gravity acceleration in the static position and the frame matrix during movement is obtained every sampling period using angular velocity. Trajectory of body segment is estimated from the numerical integration of the acceleration and angular velocity, and the joint torque is derived using Euler's equation of motion [2].

It must be careful to install the inertial sensor on the body precisely aligned to the longitudinal direction of the limbs. In this paper, the sensor axis of the WIMU are not ensured to be parallel to longitudinal axes of upper limb because the alignment procedure which estimates the relation between the sensor axes and the longitudinal and joint axes of upper limb is performed after the installation of the WIMUs on the body. To determine these relations, four preliminary behaviors are required before the pitching motion: elbow flexion-extension for the determination of the elbow 


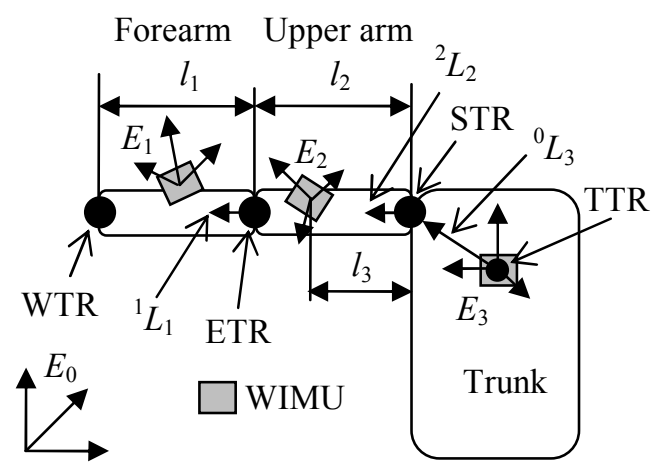

Fig. 3. Coordinate system of WIMUs and position relation of body landmark on upper limb and trunk.

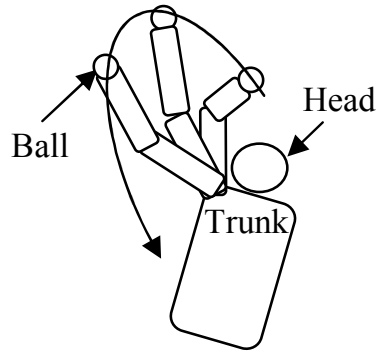

Front view

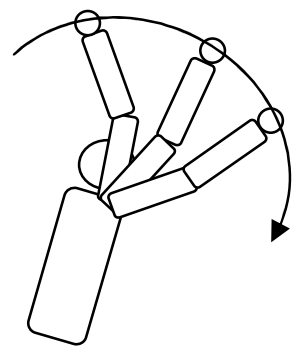

Side view (a) Overhand throwing

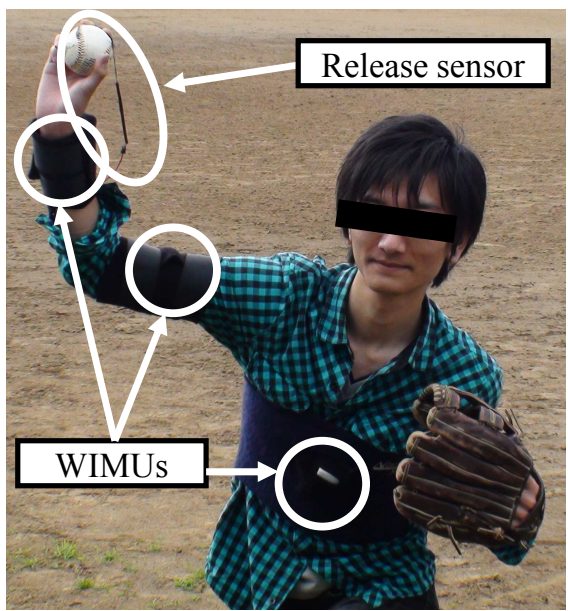

Fig. 4. Experiment scenery.

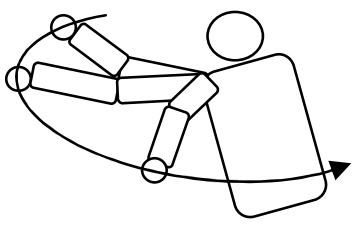

Front view

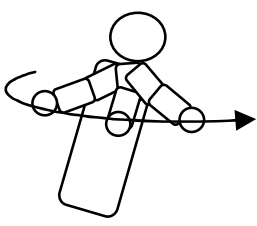

Side view

(b) Sidearm throwing

Fig. 5. Overhand throwing and sidearm throwing performed in this study.

flexion axis, shoulder adduction-abduction for elbow adduction axis, suspending the upper limb for shoulder internal rotation axis and elbow pronation one, and turn of the body around vertical and horizontal axes by keeping a set-position for the initial posture.

Because of numerical integration error, trajectories of body segments estimated by each WIMU depart steadily. Therefore, only the trajectory of the upper arm, i.e. shoulder trajectory (STR) and elbow trajectory (ETR), is calculated by acceleration integration and posture of upper arm, while wrist trajectory (WTR) and trunk trajectory (TTR) are calculated by using posture of forearm and ETR, and posture of trunk and STR, respectively. As shown in Fig. 3, the TTR, STR, ETR, and WTR are expressed as

$$
\begin{aligned}
& \mathrm{STR}=\int^{t}{ }^{0} v_{2}(\tau) d \tau-l_{3} E_{2}{ }^{2} L_{2}, \quad v(t)=\int{ }^{t}{ }^{0} a_{2}(\tau) d \tau \\
& \mathrm{ETR}=\mathrm{STR}+l_{2} E_{2}{ }^{2} L_{2} \\
& \mathrm{WTR}=\mathrm{ETR}+l_{1} E_{1}{ }^{1} L_{1} \\
& \mathrm{TTR}=\mathrm{STR}-{ }^{0} L_{3}
\end{aligned}
$$

where ${ }^{0} a_{2}$ is the upper arm acceleration based on the world coordinate system $E_{0}$. Vectors ${ }^{1} L_{1}$ and ${ }^{2} L_{2}$ are longitudinal unit vector of forearm and upper arm based on the moving coordinate system $E_{1}$ and $E_{2}, l_{1}$ and $l_{2}$ are the length of forearm and upper arm, $l_{3}$ is the distance between shoulder and WIMU on the upper arm, and ${ }^{0} L_{3}$ is the vector from chest to shoulder based on $E_{0}$.

Experimental method. One healthy male subject (age $21 \mathrm{yr}$, height $175[\mathrm{~cm}]$, weight $54[\mathrm{~kg}]$ ) participated in the experiment. Fig. 4 shows the scenery of the experiment. Three WIMUs are mounted on the forearm, upper arm and trunk using Velcro fastening tape. The subject performed the overhand throwing and the sidearm throwing as shown in Fig. 5 with 50 percent power of his 

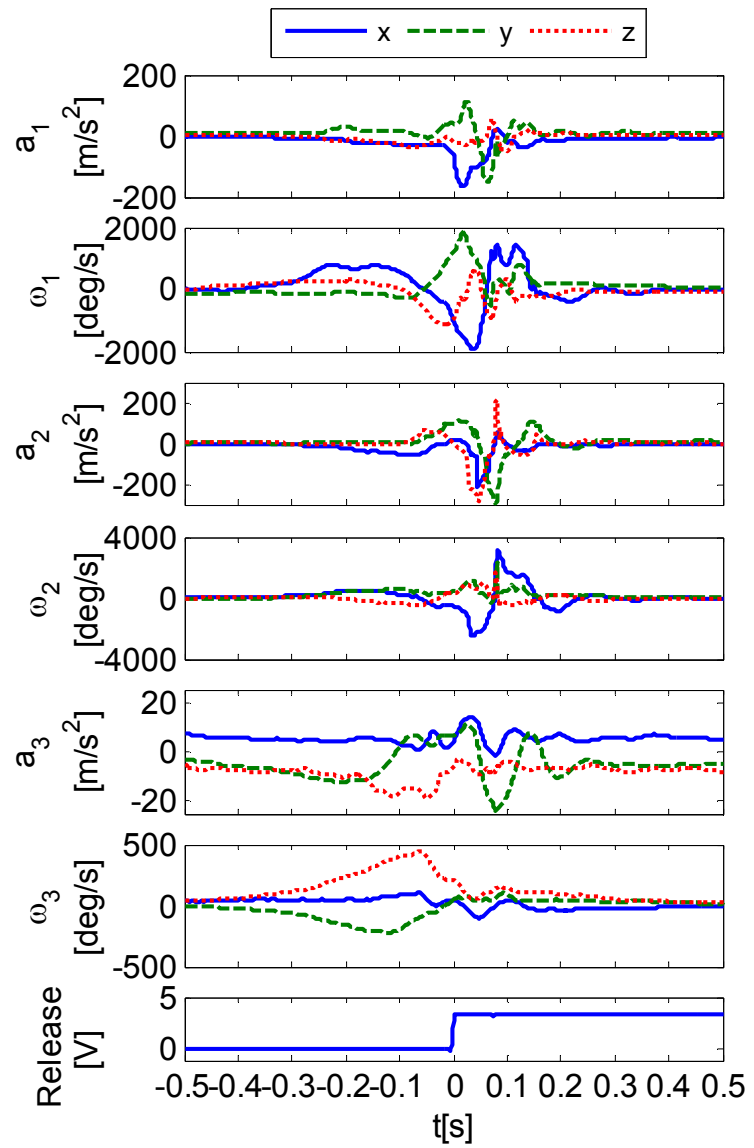

Fig. 6. An example of time trajectories of acceleration $a_{i}$ and angular velocity $\omega_{i}$ of $i$-th segment ( $i=1$ : forearm, 2 : upper arm, 3 : trunk) and output from release sensor. Release time is shifted to 0 [ms].
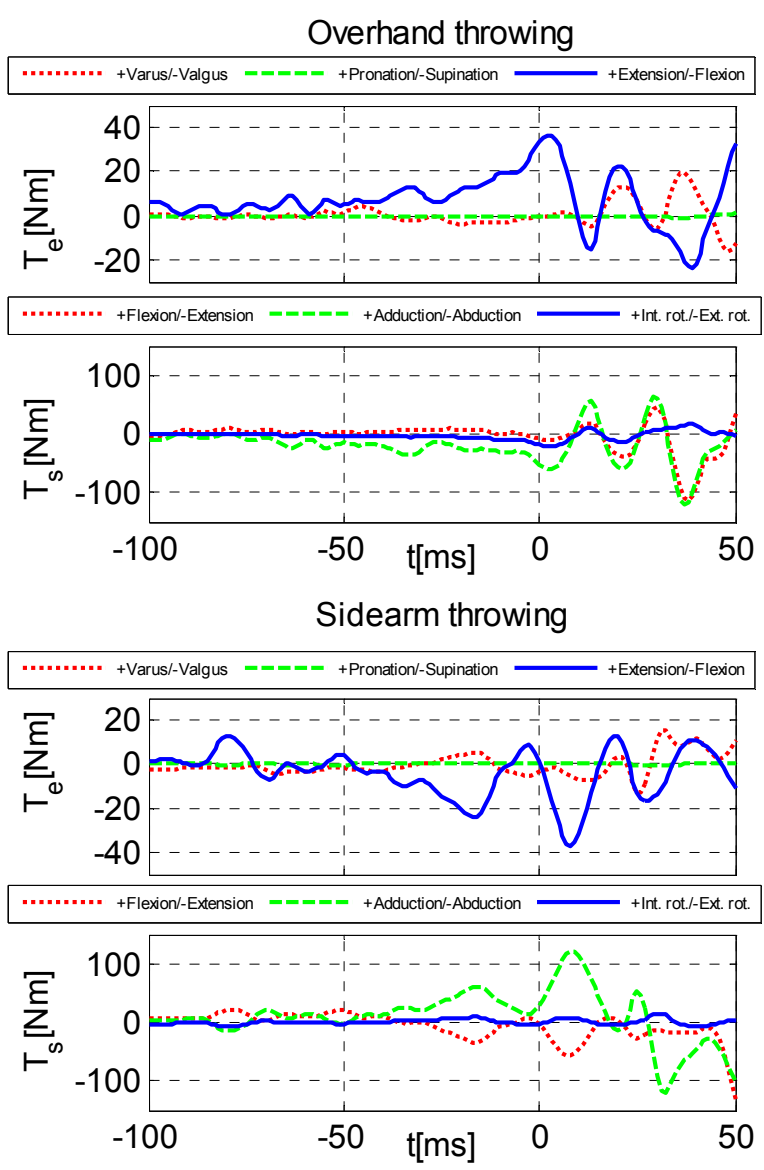

Fig. 7. Joint torque of elbow and shoulder. Notations $T_{e}$ and $T_{s}$ are elbow joint torque and shoulder joint one, respectively. Release time is shifted to 0 [ms].

maximum effort. Moreover, the subject was asked to stop moving for a few seconds before and after the pitching because the measurement of the gravitational acceleration determines the static posture. The subject understood the purpose of this study and accepted the informed consent prior to the experiment.

\section{Experimental results}

Acceleration, angular velocity and joint torque. Fig. 6 represents an example of time trajectories of acceleration $a_{i}$ and angular velocity $\omega_{i}$ of forearm, upper arm and trunk. Release time is shifted to 0 [ms] by using the output voltage from the release sensor shown in bottom of Fig.6. Because the peak of the trunk angular velocity appears earlier than that of forearm and upper arm, it is found that turning the body is occurred earlier than swinging the upper limb. Fig. 7 shows the estimated joint torque of elbow, and shoulder. In overhand throwing, the subject continued to generate shoulder abduction torque until release of the ball to keep the position of the forearm over the head. On the contrary, in sidearm throwing, the subject generated shoulder adduction torque until the release of the ball to keep the position of the arm in horizontal posture. As Fig. 7 indicates, the estimated shoulder adduction and abduction torque correspond to the difference of the pitching form. Next we give our focus to the elbow extension/flexion torque. In the overhand throwing, the elbow extension torque is increasing before the release of the ball and decreases after the ball release. This result shows that the subject released the ball when the elbow had extended, which is one of the ideal motions of the pitching. In the sidearm throwing, elbow extension torque is $9[\mathrm{Nm}]$, and it is very small compared with the overhand throwing $(36[\mathrm{Nm}])$. This shows that the elbow extension torque is not effectively 


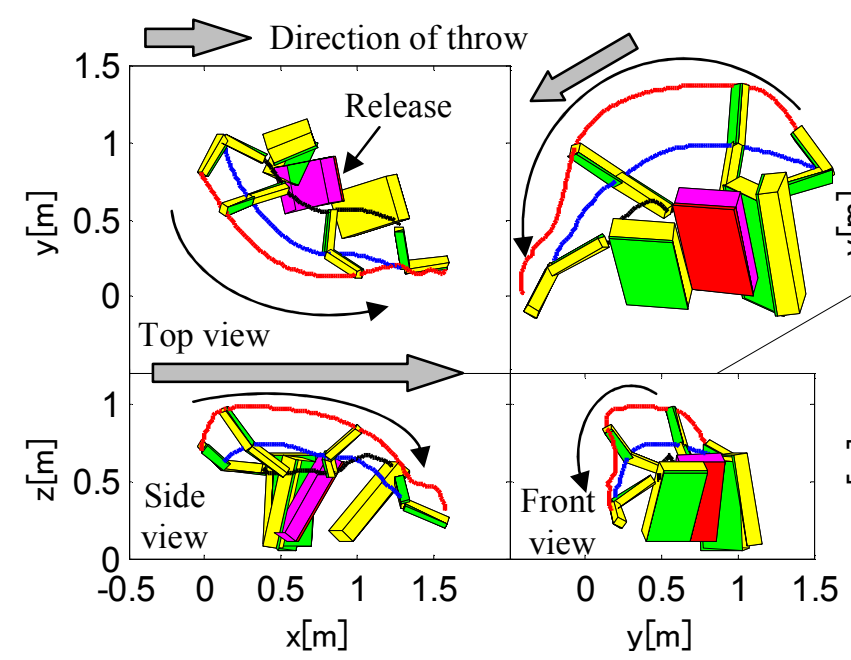

(a) Overhand throwing

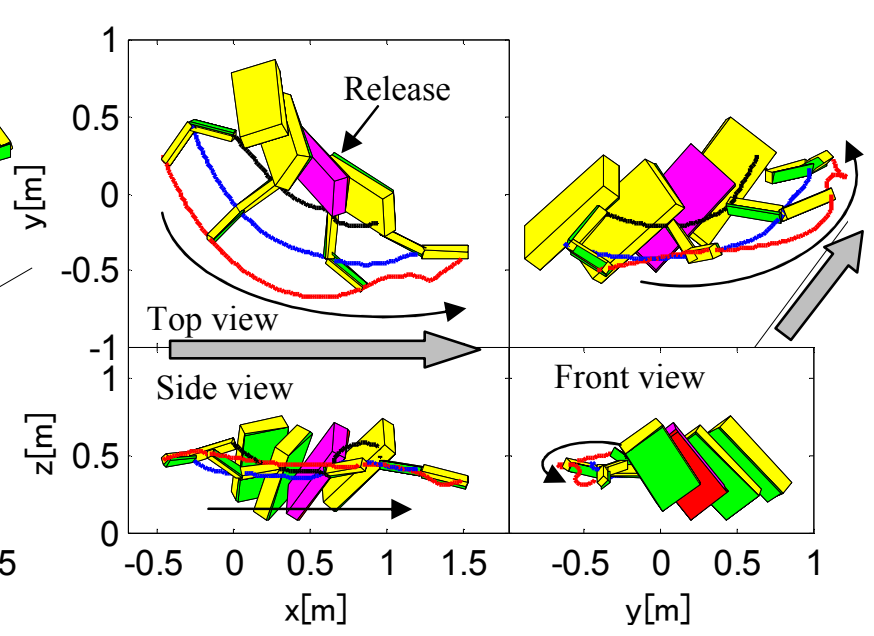

(b) Sidearm throwing

Fig. 8. Front view, side view, and top view of estimated 3D trajectory and posture. Each posture is drawn from $-200[\mathrm{~ms}]$ to 100 [ms] every 100 [ms].

used during the sidearm throwing. Detailed analysis will be performed by considering muscle torque, velocity-dependent torque, and gravity torque [3] in the future work.

3D trajectory and posture estimation. Fig. 8 shows third angle projection of 3D postures of forearm, upper arm, and trunk and their 3D trajectory when the subject performed overhand throwing (Fig.8(a)) and sidearm throwing (Fig.8(b)). As these figures indicate, 3D posture and trajectory are reconstructed corresponding to the difference of the pitching form.

From the results indicated above, it is confirmed that the proposed WIMU with small and light features can measure the 13-channel analog inputs with sampling frequency of $1000[\mathrm{~Hz}]$ of three position, record them to the microSD card, and estimate the joint torque and 3D posture of the fast movement such as baseball pitching without the limitation of the measurement place.

\section{Conclusions}

In this paper, the wireless inertial measurement unit (WIMU) for 3D pitching motion analysis has been presented. The developed WIMU prevents the users from being interfered by the connecting cables enables ubiquitous measurement. Acceleration and angular velocity during overhand and sidearm throwing are measured and 3D posture and joint torques are calculated. As the experimental results show, 3D posture qualitatively agrees with the pitching from performed, and the estimated shoulder adduction/abduction torque and elbow extension/flexion torque can be used to evaluate the pitching form. Out next study is to evaluate the whole body biomechanical efficiency of the baseball pitch using the WIMUs mounted lower limbs as well as the upper ones.

\section{References}

[1] Eric D. Parks and Tracy R. Ray, Prevention of Overuse Injuries in Young Baseball Pitchers, Sports Health: A Multidisciplinary Approach, Vol.1, No.6, 514-517, 2009.

[2] Hideharu Koda, Koichi Sagawa, Kouta Kuroshima, Toshiaki Tsukamoto, Kazutaka Urita and Yasuyuki Ishibashi, 3D Measurement of Forearm and Upper Arm during Throwing Motion using Body Mounted Sensor, Journal of Advanced Mechanical Design, Systems, and Manufacturing, Vol.4, No.1, 167-178, 2010.

[3] Masaya Hirashima, Katsu Yamane, Yoshihiko Nakamura and Tatsuyuki Ohtsuki, Kinetic chain of overarm throwing in terms of joint rotations revealed by induced acceleration analysis, Journal of Biomechanics, Vol.41, No. 13, 2874-2883, 2008. 